\title{
Análisis retrospectivo (2009-2010) de las alteraciones dermatológicas, óticas y oftalmológicas con diagnóstico clínico presuntivo de micosis en caninos $\mathbf{y}$ felinos
}

\author{
Adriana del Pilar Pulido-Villamarín ${ }^{1 *}$, Melva Yomary Linares-Linares ${ }^{1}$, Rubiela Castañeda-Salazar ${ }^{1}$, Carolina \\ Gutiérrez-Granados ${ }^{1,}$ Moisés Aranda-Silva ${ }^{2}$, Milton Januario Rueda-Varón² \\ ${ }^{1}$ Unidad de Investigaciones Agropecuarias (UNIDIA). Departamento de Microbiología. ${ }^{2}$ Departamento de Matemáticas. Facultad de Ciencias. \\ Pontificia Universidad Javeriana Bogotá, D.C. Colombia. \\ *adriana.pulido@javeriana.edu.co
}

Recibido: 29-08-2011; Aceptado: 08-11-2011

\section{Resumen}

Objetivo. Determinar la prevalencia de las alteraciones dermatológicas, óticas y oftalmológicas clínicamente presuntivas de ser causadas por hongos y analizar demográficamente su presentación. Materiales y métodos. Se realizó un estudio retrospectivo en una clínica para pequeños animales en Bogotá; se revisaron historias clínicas de pacientes que consultaron por alteraciones dermatológicas, óticas y oftalmológicas durante 2009 y 2010. Resultados. En el período evaluado la prevalencia de las alteraciones dermatológicas en caninos fue de 7,8\%, las óticas de 4,2\% y las oftalmológicas de 3,3\%. Dentro de las alteraciones dermatológicas, la sospecha etiológica principal por diagnóstico clínico fueron los dermatofitos con $86,9 \%$, en las alteraciones óticas fue Malassezia con 86,5\% y para las oftalmológicas fue Candida con $83,3 \%$. El análisis estadístico de variables no mostró asociación con los síntomas ni con los agentes etiológicos diagnosticados clínicamente; sin embargo, la evaluación categorizada evidenció asociaciones entre razas y edades con las etiologías por diagnóstico clínico. El diagnóstico de las etiologías micóticas se estableció en la mayoría de los casos por hallazgos clínicos y con menor frecuencia por raspado de piel y citología ótica; en ninguno de los casos se realizó cultivo micológico. La población felina no presentó alteraciones dermatológicas, óticas y oftalmológicas clínicamente compatibles con etiología fúngica. Conclusión. En caninos, la prevalencia de las alteraciones dermatológicas clínicamente compatibles con dermatofitos fue de 86,9\%, los procesos óticos asociados con Malassezia de 86,5\% y los oftalmológicos sospechosos de Candida con 83,3\%. Los diagnósticos definitivos se establecieron por hallazgos clínicos sin la realización de metodologías diagnósticas estándar como el cultivo.

Palabras clave: caninos, felinos, dermatofitos, Malassezia, Candida.

\begin{abstract}
A retrospective analysis (2009-2010) of the dermatological, ear and ophtalmological alterations with presumptive clinical diagnosis of mycosis in canines and felines. Objective. To determine the prevalence of dermatological, ear and ophthalmological alterations clinically presumptive to be caused by fungi and to analyze their occurrence demographically. Materials and methods. We carried out a retrospective study in a clinic in Bogota for small animals. We reviewed the medical histories of patients who requested medical appointments due to dermatological, ear and ophtalmological alterations in 2009 and 2010. Results. In the assessed period, the prevalence of dermatological alterations in dogs was of $7.8 \%, 4.2 \%$ of ear alterations and of 3.3\% of ophtalmological alterations. The main etiological suspects through clinical diagnosis were: dermatophytes with an incidence of $86.9 \%$ among dermatological alterations, Malassezia with an $86.5 \%$ of incidence in ear alterations, and Candida with $83.3 \%$ of incidence in ophtalmological alterations. Statistical analysis of variables showed no association with symptoms or clinically diagnosed etiological agents, but the categorized evaluation showed associations of races and ages with the etiologies through clinical diagnosis. The diagnosis of fungal etiologies was accomplished in most cases by clinical findings and less frequently by skin scrapings and ear cytology; in none of the cases a mycological culture was done.
\end{abstract}


The feline population showed no dermatological, ear or ophtalmological alterations compatible with a fungal etiology. Conclusion. In canines, the prevalence of dermatological alterations clinically compatible with dermatophytes was of 86.9\%; ear alterations associated with Malassezia were of $86.5 \%$; and ophtalmological alterations Candida-suspected were 83.3\%. Final diagnoses were accomplished with the help of clinical findings without following standard diagnostic methodologies such as cultivation.

Key words: canines, felines, dermatophytes, Malassezia, Candida

\begin{abstract}
Resumo
Análise retrospectiva (2009-2010) das alterações dermatológicas, óticas e oftalmológicas com diagnóstico clínico presuntivo de micose em cães e gatos. Objetivo. Determinar a prevalência das alterações dérmicas, óticas e oftálmicas clinicamente presuntivo de serem causadas por fungos e analisar demograficamente sua apresentação. Materiais e métodos. Foi realizado um estudo retrospectivo numa clínica para pequenos animais em Bogotá; foram revisadas as histórias clínicas de pacientes com alterações dérmicas, óticas e oftalmicas em 2009 e 2010. Resultados. Durante o período de avaliação a prevalência das alterações dérmicas em cães foi de 7,8\%, as óticas de 4,2\% e as oftálmicas de 3,3\%. Dentro das alterações dérmicas, a principal suspeita etiológica por diagnóstico clínico foram os dermatófitos com 86,9\%, nas alterações óticas foi Malassezia com 86,5\% e para as oftálmicas foi Candida com 83,3\%. A análise estatística das variáveis não apresentou associação com os sintomas ou com os agentes etiológicos diagnosticados clínicamente; embora, a avaliação categorizada evidenciou associações entre raças e idades com as etiologias por diagnóstico clínico. O diagnóstico das etiologias micóticas foi estabelecido na maioria dos casos pelos achados clínicos e, menos freqüentemente por raspados de pele e citologia ótica, em nenhum dos casos foi realizada uma cultura micótica. A população felina não apresentou alterações dérmicas, óticas e oftálmicas clinicamente compatíveis com etiologia fúngica. Conclusão. Em cães, a prevalência das alterações dérmicas clinicamente compatíveis com dermatófitos foi de $86,9 \%$, os processos óticos associados com Malassezia foi de 86,5\% e os oftálmicos suspeitos de Candida foi de $83,3 \%$. Os diagnósticos definitivos foram estabelecidos pelos achados clínicos sem a realização de métodos diagnósticos padroes, como o cultivo.
\end{abstract}

Palavras-chave: cães, gatos, dermatófitos, Malassezia, Candida

\section{Introducción}

Las alteraciones en la piel y anexos son un motivo de consulta común en la práctica clínica de pequeños animales; dentro de las cuales las micosis causadas por Malassezia spp., Candida spp., dermatofitos y no dermatofitos como Fusarium spp., Aspergillus spp., son las más comunes (1-3). La colonización de éstos tejidos superficiales se ve favorecida por factores predisponentes como cambio de $\mathrm{pH}$, humedad, administración prolongada de glucocorticoides o antibióticos, alteración de la barrera epitelial $(2,4-8)$ y desórdenes inmunológicos como se presenta para Candida spp. y Malassezia spp (9); constituyéndose estos últimos en un factor de riesgo para la posible diseminación sistémica $(2,10-12)$.

Los dermatofitos son un grupo de hongos que digieren la queratina del estrato córneo de piel, uñas y pelo $(6,9)$, en animales generalmente se localizan en el lomo y en la parte interna de los miembros anteriores y/o posteriores. Las lesiones se presentan como áreas escamadas, redondeadas con alopecia y no pruriginosas (6), las cuales se deben diferenciar de lesiones ocasionadas por otras etiologías. Los géneros reconocidos en las dermatofitosis son: Epidermophyton sp., Trichophyton spp. y Microsporum spp., muchos de ellos especie específica de hospederos (antropofílico, geofílico y zoofílico) $(13,14)$.
Malassezia spp., es una levadura comensal de piel y ocasionalmente patógena; $M$. pachydermatis es el agente etiológico más comúnmente asociado con dermatitis y otitis canina, aunque recientemente se han reportado otras especies. Las lesiones dermatológicas se caracterizan por alopecia, prurito con presencia de zonas hipo/hiperpigmentadas de bordes definidos, aunque no necesariamente redondeadas $(2,3)$.

Son esporádicos los reportes de otros agentes etiológicos como Candida spp., Rhodotorula spp., Trichosporum spp., Fusarium spp. Aspergillus spp. y Curvularia spp,; sin embargo, se reconoce su importancia clínica en dermatomicosis, otomicosis y queratitis $(3,15-17)$.

El diagnóstico estándar de estas infecciones, se basa en la visualización de estructuras fúngicas en las muestras clínicas y en el aislamiento de los agentes etiológicos $(4,9)$, a pesar de los falsos negativos que se pueden presentar $(4,13)$; sin embargo, en Colombia, muchas de estas alteraciones son diagnosticadas de acuerdo con la sintomatología clínica o en algunos casos solamente por citología y/o respuesta al tratamiento, lo que implica una limitación a los estudios epidemiológicos sobre las causas reales de estas alteraciones; sin embargo, como una primera aproximación y conociendo las limitaciones diagnósticas en las clínicas veterinarias se realizó un estudio retrospectivo para determinar los criterios 
de diagnóstico de enfermedades dermatológicas, óticas y oftalmológicas sospechosas de ser causadas por hongos y al mismo tiempo evaluar su prevalencia.

\section{Materiales y métodos}

\section{Recolección de datos}

Se realizó un estudio retrospectivo, en una clínica para pequeños animales ubicada en la ciudad de Bogotá, para lo cual se revisaron las historias clínicas de todos los pacientes que consultaron durante el periodo comprendido entre el 2009 y el 2010. Para determinar la prevalencia de las alteraciones dermatológicas, óticas y oftalmológicas frente a otras patologías, se tomó como parámetro de clasificación el motivo de consulta en las poblaciones canina y felina. Los datos fueron registrados en una base de datos, teniendo en cuenta variables demográficas como especie, raza, género, edad, sintomatología, diagnóstico clínico, diagnóstico por laboratorio y tratamiento. La variable raza agrupó en otras razas, las que consultaron con menor frecuencia. La variable edad se agrupó por rangos de edad en meses (1 a 12, 13 a 36,37 a 72,73 a 108, 109 a 144, 145 a 180 y mayores a 181 meses).

Para determinar la prevalencia de cada una de las alteraciones presuntivas de ser causadas por hongos, se tuvo en cuenta el diagnóstico clínico registrado en las historias de acuerdo con el criterio del médico veterinario sin el apoyo de pruebas de laboratorio.

\section{Análisis estadístico}

Se realizaron pruebas de hipótesis basadas en el índice Kappa, para determinar la asociación entre diferentes categorías de las variables de interés $(18,19)$, para lo cual se efectuaron análisis individuales, definiendo variables dicotómicas para cada categoría de respuesta. Sin embargo, paralelamente se desarrollaron las pruebas clásicas basadas en chi-cuadrado para examinar la asociación entre las variables generales, arrojando resultados similares a nivel variable. El nivel de significancia utilizado fue $\alpha=0,05$, de manera que $\mathrm{P}<0,05$ indicó una asociación estadísticamente significativa. Todas las pruebas fueron realizadas con el software estadístico $\mathrm{R}$, bajo licencia freeware.

\section{Resultados}

Durante el período comprendido entre 2009 - 2010 consultaron un total de 2099 pacientes, de los cuales 1052 $(50,2 \%)$ correspondieron al año 2009 y 1047 (49,8\%) para el 2010. Del total de los casos, el 7,8\% correspondió a alteraciones dermatológicas, el $4,2 \%$ a óticas y el 3,3\% a oftalmológicas.

Dentro de las lesiones superficiales en caninos diagnosticadas clínicamente con etiología fúngica; las alteraciones dermatológicas se asociaron a dermatofitos en un $86,9 \%$, los procesos óticos a Malassezia spp., en un 86,5\% y los oftalmológicos a Candida spp., en un $83,3 \%$ (Figura 1).

En la población felina no hubo diagnóstico clínico de procesos dermatológicos, óticos y oftalmológicos compatibles con etiología fúngica.

Los principales síntomas observados durante el examen clínico fueron prurito, alopecia, eritema, descamación, secreción y olor fétido, entre otros. Los síntomas característicos de los procesos dermatológicos y óticos se muestran en la tabla 1. Para los procesos oftalmológicos $(n=4)$ la principal sintomatología observada durante el diagnóstico clínico presuntivo de ser causado por Malassezia spp., $(n=1)$ fue secreción y para Candida spp., prurito $(n=1)$. Sin embargo, no hubo asociación entre los síntomas y la etiología diagnosticada clínicamente $(\mathrm{p}=0,235>0,05)$.

En la evaluación por razas, se evidenció que la raza French Poodle presentó la mayor frecuencia de alteraciones dermatológicas y óticas compatibles con afección por Malassezia spp., siendo ésta última la que mostró asociación estadísticamente significativa $(\mathrm{p}=0,00<0,05)$; en la raza Boxer solamente hubo asociación entre alteraciones dermatológicas y los dermatofitos $(\mathrm{p}=0,004<0,05)$, mientras la raza criollo presentó asociación de los procesos óticos con Malassezia spp., ( $\mathrm{p}=0,009$ < 0,05) (Tabla 2). En el grupo de otras razas, Candida spp., fue el principal diagnóstico clínico presuntivo asociado con alteraciones dermatológicas $(100 \%)$, óticas $(100 \%)$ y oftalmológicas (75\%). De igual forma, en la raza Schnauzer los procesos oftalmológicos, se asociaron a Malassezia spp., en el 100\% de los casos. Sin embargo, aunque se observaron algunos valores p estadísticamente significativos, estos resultados no permitieron realizar inferencias, debido al bajo número de observaciones (n).

Frente a la variable edad, en caninos menores de un año se observó mayor presentación de alteraciones dermatológicas y óticas (Tabla 3), donde se determinó asociación estadísticamente significativa frente a diagnósticos clínicos por dermatofitos $(\mathrm{p}=0,002)$ y Malassezia spp., $(\mathrm{p}=0,028)$. Aunque se observó asociación de la edad y los procesos dermatológicos presuntivos de Malassezia spp., en el grupo de 145 a 180 meses $(\mathrm{p}=0,00)$, ésta no se consideró por tener un bajo valor de n. La misma observación se hizo 


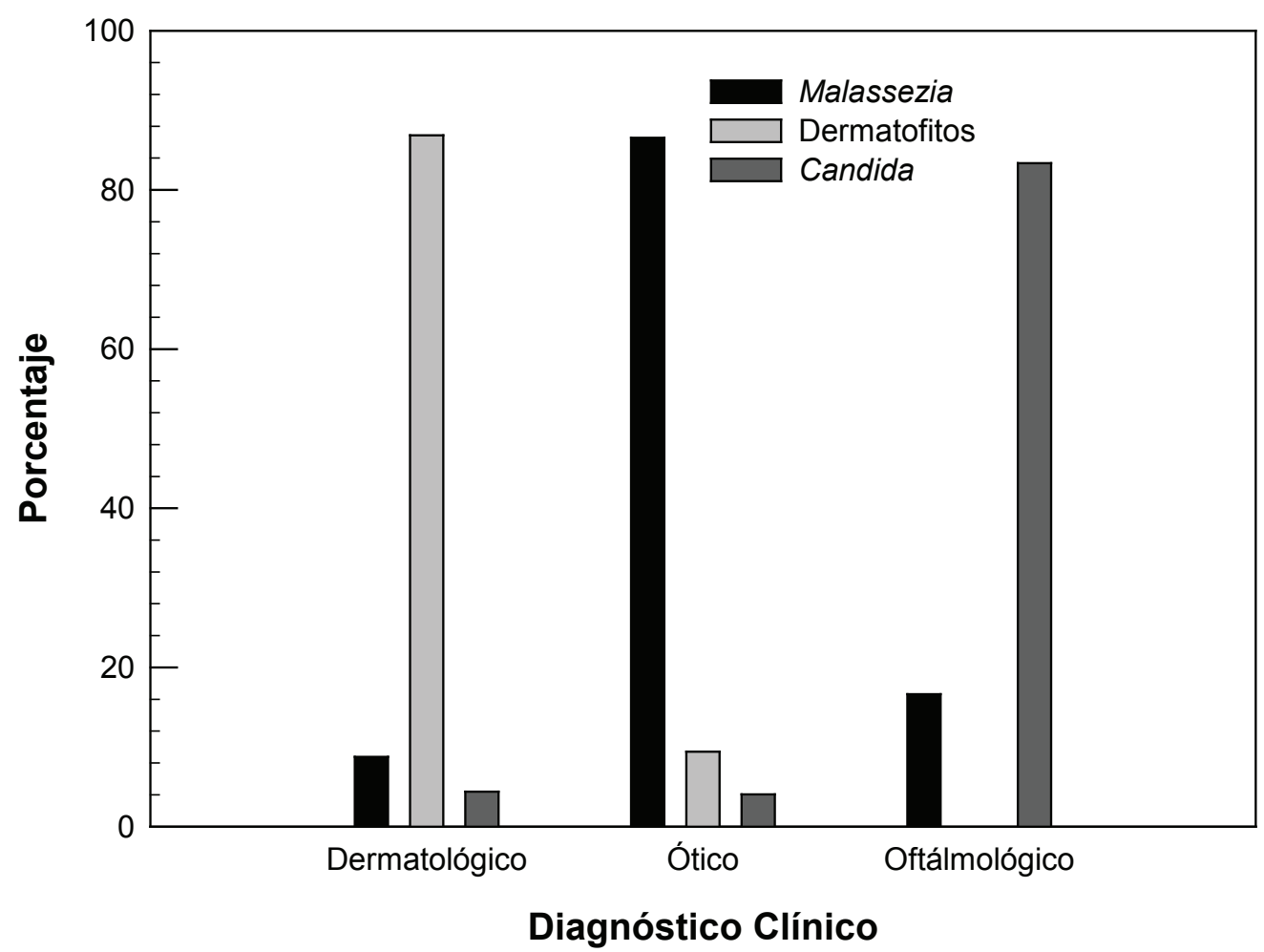

Figura 1. Distribución etiológica por diagnóstico clínico en población canina (2009-2010).

Tabla 1. Sintomatología según etiología por diagnóstico clínico

\begin{tabular}{lcccccc}
\hline & \multicolumn{3}{c}{ Dermatológico $\mathbf{N}=\mathbf{4 6}$} & \multicolumn{3}{c}{ Ótico $\mathbf{N}=\mathbf{5 5}$} \\
\cline { 2 - 7 } \multicolumn{1}{c}{ Sintomatología } & 1 & 2 & 3 & 1 & 2 & 3 \\
\cline { 2 - 7 } & $\mathrm{n}=4$ & $\mathrm{n}=40$ & $\mathrm{n}=2$ & $\mathrm{n}=48$ & $\mathrm{n}=5$ & $\mathrm{n}=2$ \\
\cline { 2 - 6 } & - & 15,0 & - & - & - & - \\
Alopecia & - & 7,5 & - & 14,9 & - & - \\
Prurito & - & - & - & 23,4 & 20,0 & 50,0 \\
Secreción & - & 2,5 & - & - & - & - \\
Descamación & - & - & - & 6,4 & - & - \\
Eritema & 75,0 & 52,5 & 100,0 & 25,5 & 60,0 & 50,0 \\
Mixtas & 25,0 & 22,5 & - & 29,8 & 20,0 & - \\
Sin dato & & & & & & \\
\end{tabular}

1: Malassezia. 2: Dermatofitos. 3: Candida.

Mixta (alopecia y/o prurito y/o secreción y/o olor fétido y/o descamación y/o eritema). 
Tabla 2. Razas según etiología por diagnóstico clínico.

\begin{tabular}{|c|c|c|c|c|c|c|}
\hline \multirow{4}{*}{ Raza } & \multicolumn{3}{|c|}{ Dermatológico N= 46} & \multicolumn{3}{|c|}{ Ótico $\mathrm{N}=55$} \\
\hline & 1 & 2 & 3 & 1 & 2 & 3 \\
\hline & $\mathrm{n}=4$ & $\mathrm{n}=40$ & $\mathrm{n}=2$ & $\mathrm{n}=48$ & $n=5$ & $\mathrm{n}=2$ \\
\hline & \multicolumn{6}{|c|}{$\%$} \\
\hline Beagle & - & 13,0 & - & 4,2 & 20,0 & - \\
\hline Boxer & - & $13,0 *$ & - & - & - & - \\
\hline French Poodle & 25,0 & 20,0 & - & $52,1 *$ & 40,0 & - \\
\hline Gold Retriever & - & - & - & 8,3 & $20,0 *$ & - \\
\hline Labrador & - & 7,0 & - & 4,2 & 20,0 & - \\
\hline Schnauzer & - & - & - & 6,3 & - & - \\
\hline Pastores & - & - & - & 2,1 & - & - \\
\hline Cocker Spaniel & - & 5,0 & - & 2,0 & - & - \\
\hline Criollo & $50,0 *$ & 12,0 & - & $4,2 *$ & - & - \\
\hline Otros & - & 10,0 & 100,0 & 2,1 & - & 100,0 \\
\hline Sin Dato & 25,0 & 20,0 & - & 14,6 & - & - \\
\hline
\end{tabular}

Malassezia. 2: Dermatofitos. 3: Candida

Otros: razas que consultaron con menor frecuencia.

$* \mathrm{p}<0,05$

Tabla 3. Rangos de edad según etiología por diagnóstico clínico

\begin{tabular}{|c|c|c|c|c|c|c|}
\hline \multirow{4}{*}{ Edad } & \multicolumn{3}{|c|}{ Dermatológico $N=46$} & \multicolumn{3}{|c|}{ Ótico $\mathrm{N}=55$} \\
\hline & 1 & 2 & 3 & 1 & 2 & 3 \\
\hline & $\mathrm{n}=4$ & $\mathrm{n}=40$ & $\mathrm{n}=2$ & $\mathrm{n}=48$ & $\mathrm{n}=5$ & $\mathrm{n}=2$ \\
\hline & \multicolumn{6}{|c|}{$\%$} \\
\hline 1-12 meses & 25,0 & $20,0^{*}$ & 100,0 & $54,2 *$ & 20,0 & 100,0 \\
\hline 13-36 meses & - & 30,0 & - & 15,0 & $40,0 *$ & - \\
\hline 37-72 meses & - & 12,5 & - & 7,5 & - & - \\
\hline 73-108 meses & - & 2,5 & - & 10,0 & $40,0 *$ & - \\
\hline 109-144 meses & - & - & - & 5,0 & - & - \\
\hline $145-180$ meses & $50,0^{*}$ & 5,0 & - & - & - & - \\
\hline$>181$ meses & - & - & - & - & - & - \\
\hline Sin dato & 25,0 & 3,0 & - & 17,5 & - & - \\
\hline
\end{tabular}

1: Malassezia. 2: Dermatofitos. 3: Candida

$* \mathrm{p}<0,05$ 
respecto a los procesos óticos compatibles con dermatofitos (13-36 y 73-108 meses) (Tabla 3). A pesar de no presentar asociación estadísticamente significativa, las alteraciones oftalmológicas compatibles clínicamente con Malassezia spp., y Candida spp., afectaron en el $100 \%$ a cachorros entre 1 a 12 meses.

Para la variable género, las alteraciones dermatológicas en machos fueron del 59,8\% y para las hembras del 38,2\%. En las alteraciones óticas el porcentaje fue similar para los dos géneros, 54,2 \% y 45,0\% respectivamente; así como para las alteraciones oftalmológicas, 51,0\% en machos y 45,3\% en hembras. Los porcentajes restantes corresponden a pacientes donde el dato del género no se encontraba registrado.

Las pruebas de laboratorio realizadas para complementar el diagnóstico clínico de las alteraciones dermatológicas fueron raspados de piel en un 76,3\% y para los procesos óticos citología en un $77,6 \%$ mientras que para los procesos oftalmológicos no se realizó ninguna prueba diagnóstica adicional; sin embargo, en ninguno de los casos se establecieron diagnósticos definitivos por cultivos micológicos para determinar la etiología exacta del proceso clínico (Figura 2).

Los tratamientos farmacológicos de los procesos dermatológicos y óticos se registran en la tabla 4. Los azoles (Ketoconazol, Itraconazol, Miconazol y Clotrimazol) fueron la terapia utilizada en los procesos dermatológicos presuntivos de Malassezia spp., (75\%) y dermatofitos (52,5\%), mientras que para los procesos óticos no se estableció una terapia específica. En los procesos oftalmológicos se utilizaron antibióticos en el 100\% de los casos asociados a Malassezia spp., y para los casos presuntivos de Candida spp., se utilizaron azoles en $33,3 \%$

\section{Discusión}

La frecuencia de consultas registradas durante el 2009 y el 2010 no muestra diferencias por año; sin embargo, se puede observar que hay una mayor consulta de la población canina $94,5 \%$ frente a población felina 5,5\%. Los motivos de consulta que presentaron mayor prevalencia fueron las
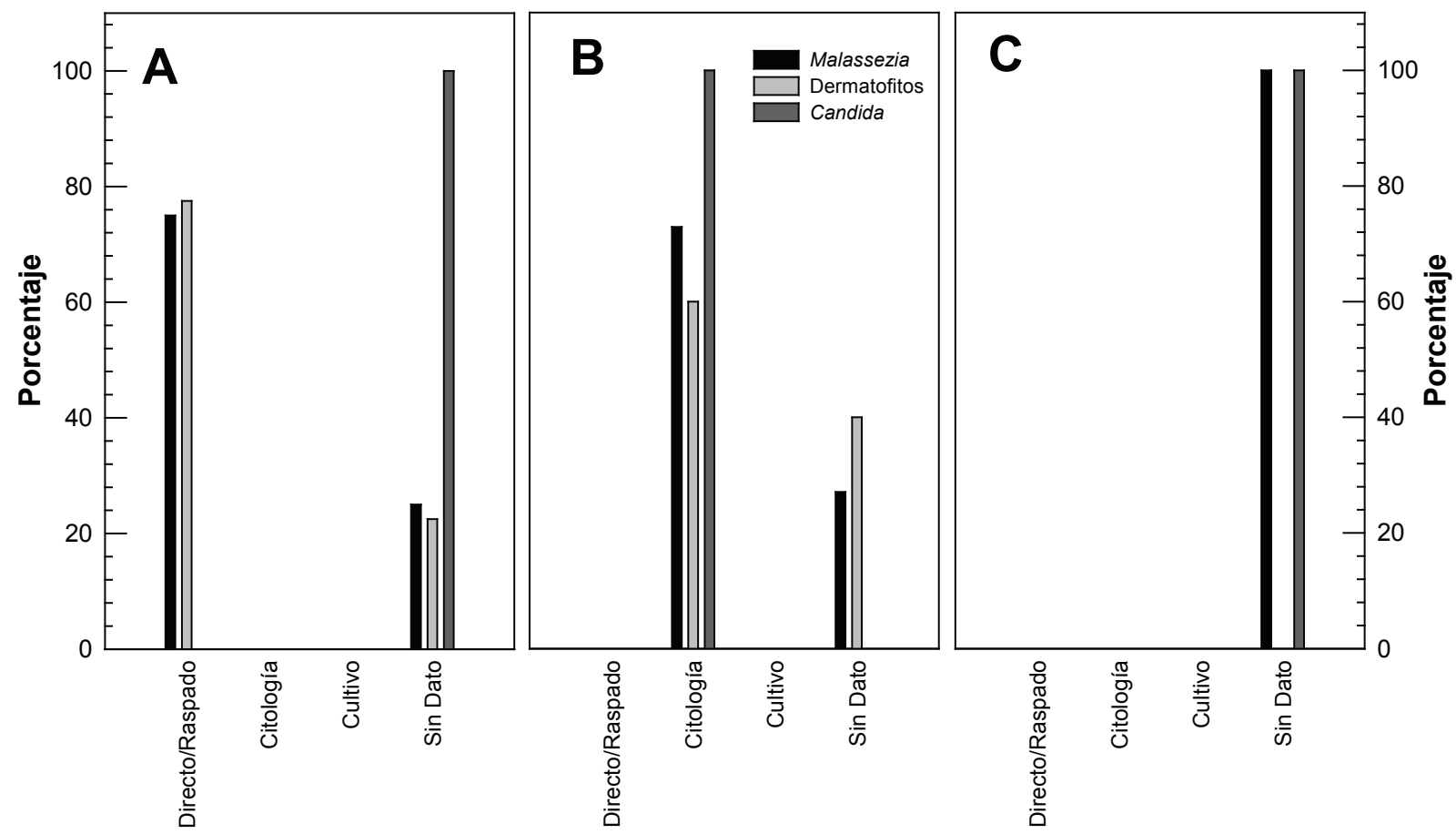

\section{Prueba diagnóstica}

Figura 2. Pruebas de laboratorio complementarias para el diagnóstico clínico. (Panel A: Dermatológico. Panel B: Ótico. Panel C: Oftalmológico) 
Tabla 4. Tratamiento según etiología por diagnóstico clínico

\begin{tabular}{lcccccc}
\hline & \multicolumn{3}{c}{ Dermatológico } & \multicolumn{3}{c}{ Ótico } \\
\cline { 2 - 7 } Tratamiento & 1 & 2 & 3 & 1 & 2 & 3 \\
\cline { 2 - 7 } & $\mathrm{n}=4$ & $\mathrm{n}=40$ & $\mathrm{n}=2$ & $\mathrm{n}=48$ & $\mathrm{n}=5$ & $\mathrm{n}=2$ \\
\cline { 2 - 7 } Griseofulvina & - & 10,0 & - & - & - & - \\
*Azoles & & 52,5 & - & 29,2 & - & - \\
*Antibiótico & - & 2,5 & - & - & - & - \\
Otros & 25,0 & 22,5 & 100,0 & 64,5 & 60,0 & - \\
Sin dato & 25,0 & 12,5 & - & 6,3 & 40,0 & 100 \\
\hline
\end{tabular}

1: Malassezia. 2: Dermatofitos. 3: Candida.

* Antibiótico o antifúngico solo o en combinación con corticoide y analgésico.

Otros:(vitaminas,antiparasitarios, analgésicos, antihistamínicos, baños medicados.

alteraciones gastroenterológicas (dato no mostrado) y dermatológicas en la población canina, mientras que en felinos, el principal motivo de consulta fue por traumas y problemas gastroenterológicos (datos no mostrados), seguidos de procesos dermatológicos, aunque ninguno de ellos asociado clínicamente con etiología fúngica.

Las micosis superficiales causadas por dermatofitos y levaduras como Malassezia spp., y Candida spp., son importantes en alteraciones dermatológicas de perros y gatos $(3,6,9)$, sin embargo, en este estudio no se evidenció alteraciones compatibles con micosis en población felina donde se reportan prevalencias por dermatofitos entre $20-30 \%(5,7)$.

Dentro de las alteraciones superficiales causadas por hongos en población canina, los procesos dermatológicos se asociaron por clínica principalmente a dermatofitos en un $86,9 \%$ de los casos, de los cuales al $77,5 \%$ se les realizó raspado de piel y en ninguno de ellos se realizó cultivo. Estos resultados difícilmente se pueden comparar con lo reportado en la literatura, ya que algunos estudios mediante microscopía directa y cultivo reportan un 86,2\% de asociación a etiología fúngica, donde solo el 33,7\% corresponde a dermatofitos $(4,15,16)$, aunque otros autores muestran prevalencias bajas que oscilan entre $4 \%$ y $20 \%(5-7,20,21)$. Es importante resaltar que los datos obtenidos en este estudio retrospectivo se basan en su mayoría por hallazgos clínicos sin una confirmación diagnóstica mediante cultivo, por lo que la prevalencia aquí reportada podría variar considerablemente. En Colombia, se desconocen epidemiológicamente las especies asociadas a las dermatofitosis en caninos; sin embargo, a nivel mundial la literatura reporta a Microsporum canis como el principal agente etiológico de estos procesos (4, 9, 21-24).

La prevalencia general de las alteraciones óticas ha sido reportada en un rango del 5 - 20\% (8, 26). El género Malassezia, es uno de los microorganismos que en pequeñas especies ha sido reportada en procesos dermatológicos y óticos en un 50-83\%, en promedio para las dos patologías $(25,26)$. En el presente estudio, del 4,2\% de las alteraciones óticas, el 86,5\% fueron clínicamente diagnosticadas como otitis externa por Malassezia, de los cuales solo al 72,9\% se les realizó análisis citológico, prueba que no permite por características morfológicas una fácil diferenciación entre géneros levaduriformes (27). En Colombia, no se conocen datos precisos de las especies de Malassezia implicadas en procesos óticos, sin embargo, por estudios previos realizados en el grupo de investigación, se reportó para otitis externa una prevalencia del 12,4\% para Malassezia furfur, 32,2\% para $M$. pachydermatis y 52,1\% para Malassezia spp., etiologías confirmadas mediante citología y cultivo (28).

Los procesos oftalmológicos se presentaron en un 3,5\% de los casos, dentro de la cuales el $83.3 \%$ se atribuyó clínicamente a Candida spp., y en menor proporción a Malassezia spp., $(16,7 \%)$. Son pocos los reportes de patologías oftalmológicas asociadas a Candida spp., en pequeñas especies, sin embargo, se ha reportado ocasionalmente en conjuntiva de bovinos, como flora normal de mucosas en caninos y como patógeno en animales inmunosuprimidos a nivel de mucosa oral, respiratoria, genitourinaria y ocular (29-31). Aunque las alteraciones oftalmológicas que se presentan con mayor frecuencia son las de tipo bacteriano, Malassezia 
pachydermatis ha sido reportada en un $3 \%$ en perros sanos y en un $23 \%$ en perros con úlcera corneal (32).

Para las alteraciones dermatológicas, óticas y oftalmológicas predominó la sintomatología mixta en las tres etiologías evaluadas (Tabla 1), sin embargo, otros autores han reportado casos con costras, pápulas y pústulas foliculares $(1,2$, 33 ), sintomatología que no fue reportada en este estudio. Otros signos asociados con estos procesos son eritema, liquenificación con o sin hiperpigmentación, escoriaciones, seborrea y prurito $(2,5-7,33-35)$.

El análisis de las alteraciones dermatológicas, óticas y oftalmológicas frente a las variables demográficas, demostró que la mayoría de procesos dermatológicos en población canina se presentó entre 1 y 36 meses, lo que coincide con lo reportado por diferentes autores, quienes determinan una prevalencia de procesos dermatológicos y óticos mayor en animales jóvenes, enfermos o débiles (1, $3,5-7,36)$, posiblemente debido a que su sistema inmune es inmaduro o porque fisiológicamente no producen ácido linoléico que actúa como compuesto fungistático $(5,7)$; sin embargo, autores como Dragonetti y Broglia (2007) (26) afirman que los pacientes más susceptibles a estas patologías se encuentran entre los 60 a 96 meses, por considerarse un período geriátrico donde la capacidad de respuesta inmune se encuentra disminuida (1); sin embargo, otros autores afirman que la edad no juega un papel importante en la presentación de las mismas (9). En este estudio las alteraciones óticas se presentaron en todos los rangos de edad, aunque los reportes muestran altas prevalencias de otitis externas en caninos jóvenes (8).

Frente a la variable género, clínicamente no hubo asociación entre las alteraciones superficiales y los agente etiológicos evaluados, a pesar de la mayor afluencia de consultas por machos con procesos dermatológicos y óticos, lo que concuerda con autores que afirman que el género no se considera un factor predisponente para la presentación de estas alteraciones $(1,7,25,37,38)$; sin embargo, Cafarchian y colbs. (2004) (5) y Prado y colbs. (2008) (9), reportan que los machos pueden tener una mayor predisposición, debido a factores de tipo hormonal o diferencias en la composición del contenido lipídico.

En relación con la raza, se observó asociación entre la raza Boxer con procesos dermatológicos compatibles con dermatofitos y entre las razas French poodle y criollo con procesos óticos asociados clínicamente a Malassezia ( $p<0,05)$; sin embargo, se ha observado que la contaminación de animales por hongos geofílicos se ve favorecida en razas con pelo largo o por la composición del contenido lipídico de la piel $(5,6)$. En cuanto a las alteraciones óticas, la raza
French poodle tuvo una prevalencia alta de otitis con etiología compatible por dermatofitos $(40,0 \%)$ y por Malassezia $(52,1 \%)$, raza que se encuentra asociada a estas etiologías por disposición de oreja pendulosa y por la cantidad de pelo en el conducto auditivo (8).

Como apoyo al diagnóstico clínico de las micosis superficiales en los procesos dermatológicos y óticos se realizaron raspados de piel en un $76,3 \%$ y citología ótica en un 77,6\% respectivamente; en ningún caso se estableció diagnóstico definitivo por cultivo micológico, lo que hace difícil el conocer epidemiológicamente las especies que circulan en nuestro medio. A pesar de los resultados registrados en las historias clínicas, no son claros los parámetros evaluados para definir la etiología fúngica reportada. El diagnóstico para dermatofitos en muchos casos se basó únicamente en las características de las lesiones, lo que es poco confiable, siendo el cultivo la prueba de oro, que permite la identificación completa del agente etiológico, llevando a un diagnóstico definitivo y preciso $(1,2,4,33)$. En los procesos óticos asociados a Malassezia el diagnóstico se basó principalmente en la citología, sin embargo, teniendo en cuenta que esta levadura se constituye como flora comensal es importante una adecuada interpretación basada en antecedentes, hallazgos clínicos, resultados citológicos y cultivo (2, 27, $33,34,39)$. Se debe tener en cuenta que para establecer un diagnóstico preciso es necesario considerar la sensibilidad y especificidad de las pruebas utilizadas, ya que a pesar de que la citología permite evidenciar estructuras micóticas es necesario complementar el diagnóstico por otras técnicas como cultivo y serología entre otras $(4,9,40)$.

En cuanto al tratamiento, los azoles (Ketoconazol, Itraconazol, Miconazol y Clotrimazol) fueron la terapia utilizada en los procesos dermatológicos compatibles con Malassezia (75\%) y con dermatofitos (52.5\%). Sin embargo, llama la atención que para los procesos óticos asociados a Malassezia y dermatofitos no se estableció una terapia antifúngica sino que su manejo estuvo enfocado a disminuir la inflamación y a realizar limpieza del conducto auditivo. Aunque es difícil establecer una estrategia terapéutica única para el tratamiento de los dermatofitos, se sugiere el uso de antimicóticos como griseofulvina, itraconazol, terbinafina, ketoconazol, fluconazol $(1,33)$ y enilconazole como las más efectivas $(1,2)$; sin dejar de lado la limpieza rigurosa del hábitat de los animales $(2,12,23,33,41,42)$. Por otro lado, el tratamiento indicado para Malassezia sugiere terapia tópica con miconazol, ketoconazol, clotrimazol, enilconazol, nistatina o clorhexidina, a los que $M$. pachydermatis es susceptible. En casos crónicos o generalizados se recomienda terapia sistémica con ketoconazol, itraconazol o fluconazol $(2,34,39,40)$. 
Para los procesos oftalmológicos, en el $100 \%$ de los casos que se asociaron a Malassezia se utilizaron antibióticos, mientras que para los compatibles con Candida se utilizaron azoles en un $33,3 \%$ y otros medicamentos en el $66,7 \%$; sin embargo, reportes recientes han evidenciado que la gentamicina en altas concentraciones puede inhibir el crecimiento de $M$. pachydermatis in vitro, lo que sugiere que el tratamiento con este antibiótico podría ser eficaz en casos de patologías por Malassezia (43).

\section{Conclusiones}

En el presente estudio, la prevalencia de los diagnósticos que se asociaron clínicamente a procesos dermatológicos causados por dermatofitos fueron de $86,9 \%$, los procesos óticos por Malassezia spp., de 86,5\% y los oftalmológicos por Candida de $83,3 \%$.

Basados en estudios epidemiológicos se ha visto que la presentación de alteraciones superficiales por hongos tienden a aumentar con el tiempo $(1,6,9,21)$, por lo que a nivel nacional se requiere confirmar los diagnósticos por medio de pruebas de laboratorio que incluyan la identificación de género y especie mediante cultivo, de manera que el conocimiento epidemiológico de estas patologías sea oportuno y preciso; además de reconocer la importancia zoonótica de la mayoría de estos procesos $(3,6,7)$.

\section{Agradecimientos}

A la Clínica Veterinaria ABANIMAL, al Dr. Daniel Navarrete y a todo el personal que labora en la institución.

\section{Financiación}

Este estudio restrospectivo fue realizado con recursos propios de la Unidad de Investigaciones Agropecuarias - UNIDIA-

\section{Conflicto de intereses}

Los autores declaran que no existe conflicto de intereses en la publicación del presente documento.

\section{Referencias}

1. Moriello KA. Treatment of dermatophytosis in dogs and cats: review of published studies. Veterinary Dermatology 2004; 15, 99-107.

2. Outerbridge A. Mycologic disorders of the skin. Topics in Companion Animal Medicine 2006; 21 (3): 128-134.
3. Bond R. Superficial veterinary mycoses. Clinics in Dermatology 2010; 28, 226-236.

4. Nichita I, Marcu A. The fungal microbiota isolated from cats and dogs. Animal Science and Biotechnologies 2010; 43 (1): 411-414.

5. Cafarchian C, Romito D, Sasanelli M, Lia L, Capelli G, Otranto D. The epidemiology of canine and feline dermatophytoses in southern Italy. Mycoses 2004; 47, 508-513.

6. Chermette R, Ferreiro L, Guillot J. Dermatophytoses in Animals. Mycopathologia 2008; 166, 385-405.

7. Seker E, Dogan N. Isolation of dermatophytes from dogs and cats with suspected dermatophytosis in Western Turkey. Preventive Veterinary Medicine 2011; 98, 46-51.

8. Zur G, Lifshitz B, Bdolah-Abram T. The association between the signalment, common causes of canine otitis externa and pathogens. Journal of Small Animal Practice 2011; 52, 254-258.

9. Prado MR, Brilhante RSN, Cordeiro RA, Monteiro AJ, Sidrim JJC, Rocha NFG. Frequency of yeasts and dermatophytes from healthy and diseased dogs. Journal Veterinary Diagnostic Invest 2008; 20, 197-202.

10. Pérez V, Corpas J, García Marín J, Adúriz J, Jensen H. Mammary and systemic aspergillosis in diary sheep; Veterinary Pathology 1998; 35, 235-240.

11. Pérez J, Carrasco L. Diagnóstico histopatológico de micosis en patología veterinaria, Revista Iberoamericana de Micología 2000; 17, S18-S22.

12 Venturini C, Morais S, Sydnei C, Aurea B, Siqueira A, Canabarro A, Hartz A. Dermatophytes isolated from dogs and cats suspected of dermatophytosis in Southern Brazil, Acta Scientiae Veterinariae 2006; 34 (2): 119-124.

13. Ross B. Superficial veterinary mycoses. Clinics in Dermatology 2010; 28, 226-236.

14. Lorio R, Cafarchia C, Capelli G, Fasciocco D, Otranto D, Giangaspero A. Dermatophytoses in cats and humans in Central Italy: epidemiological aspects. Mycoses 2007; 50, 491-495.

15. Qureshi S, Wani SA, Beg S. Curvularia dermatomycosis in a jersey heifer: a case report. Pakistan Veterinary Journal 2006; 26 (3): 149-150.

16. Coyner K. Otomycosis due to Aspergillus spp. in a dog: case report and literature review. Veterinary Dermatology 2010; 21, 613-618.

17. Ghibaudo G, Peano A. Chronic monolateral otomycosis in a dog caused by Aspergillus ochraceus. Veterinary Dermatology 2010; 21, 522-526. 
18. Bartfay E, Donner A. The effect of collapsing multinomial data when assessing agreement. International Journal of Epidemiology 2000; 29: 1070-1075.

19. Hu LP, Bao XL, Tao LX. Statistical inference and experimental design of qualitative data of paired design. Journal of Chinese Integrative Medicine 2011; 9 (1): 109-112.

20. Khosravi A, Mahmoudi M. Dermatophytes isolated from domestic animals in Iran. Mycoses 2003; 46, 222-225.

21. Mancianti F, Nardoni S, Corazza M, D’Achille P, Ponticelli C. Environmental detection of Microsporum canis arthrospores in the households of infected cats and dogs. Journal of Feline Medicine and Surgery 2003; 5, 323-328.

22. Cabañes F, Abarca M, Bragulat M. Dermatophytes isolated from domestic animals in Barcelona, Spain. Mycopathologia 1997; 137, 107-113.

23. Cabañes F. Dermatofitosis animales. Recientes avances. Revista Iberoamericana de Micología 2000; 17, S8-S12.

24. Segundo C, Martínez A, Arenas R, Ramón F, Cervantes R. Dermatomicosis por Microsporum canis en humanos y animales. Revista Iberoamericana de Micología 2004; 21, 39-41.

25. Khosravi AR, Eid S, Ziglari T, Bayat M. Isolation and Differentiation of Malassezia Species Isolated from Healthy and Affected Small Animals, Ear and Skin. World Journal of Zoology 2008; 3 (2): 77-80.

26. Dragonetti A, Broglia G. Otitis externa canina aproximación al diagnóstico. Universidad Nacional de la Plata. Veterinaria Cuyana 2007; 1, 28-33.

27. Ginel PJ, Lucena R, Rodriguez JC, Ortega J. A semiquantitative cytological evaluation of normal and pathological samples from the external ear canal of dogs and cats. Veterinary Dermatology 2002; 13, 151-156.

28. Pulido VAP, Castañeda SR, Linares LM, Mercado RM. Diagnóstico clínico microbiológico de otitis externa en caninos de Bogotá-Colombia. Revista MVZ Córdoba. 2010; 15 (3): 2215-2222.

29. Sgorbini M, Barsotti G, Nardoni S, Brombin M, Sbrana A, Mancianti F, Corazza M. Seasonal prevalence of fungi in the conjunctival fornix of healthy cows during a 2-year study. Veterinary Ophthalmology 2010; 13 (4): 227-234.

30. Brito EHS, Fontenelle ROS, Brilhante RSN, Cordeiro RA, Monteiro AJ, Sidrim JJC, Rocha MFG. The anatomical distribution and antimicrobial susceptibility of yeast species isolated from healthy dogs. The Veterinary Journal 2009; 182, 320-326.
31. Greene CE. Dermatofitosis En: Greene CE. Enfermedades infecciosas en perros y gatos. $2^{\mathrm{a}}$ Edición. McGraw-Hill Interamericana. México D.F. México.2000; 1014p.

32. Prado MR, Brito EHS, Girão MD, Monteiro AJ, Sidrim JJC, Rocha MFG. Higher incidence of Malassezia pachydermatis in the eyes of dogs with corneal ulcer than in healthy dogs. Veterinary Microbiology 2004; 100, 115-120.

33. Foil CS. Dermatofitosis En: Greene CE. Enfermedades infecciosas en perros y gatos. $2^{\text {a }}$ Edición. McGraw-Hill Interamericana México D.F. México 2000; 1014p.

34. Patterson AP, Frank LA. How to diagnose and treat Malassezia dermatitis in dogs. Veterinary Medicine 2002; 612-622.

35. Hill P, Rybnícek J, Lau-Gillard P. Correlation between pruritus score and grossly visible erythema in dogs. Veterinary Dermatology 2010; 21, 450-455.

36. Sanjuán M, Corrales J, Yus E. Prevalencia de diversas enfermedades infecciosas en un colectivo canino con un estado sanitario deficiente. Journal de Medicina Veterinaria. 2003; 20 (5): 55-61.

37. Egenvall A, Hedhammar Ä, Bonnett B. Gender, age and breed pattern of diagnoses for veterinary care in insured dogs in Sweden during 1996. Veterinary Record 2000; 146, 551-557.

38. Crespo M, Abarca M, Cabañes F. Ocurrence of Malassezia sp. in the external ear canals of dog and cats with and without otitis externa. Medical Mycology 2002; 40, 115-121.

39. Rejas LJ. Dermatitis canina por Malassezia. Revista Electrónica de Veterinaria. 2009; 9 (5): 1-13.

40. Cornegliani L, Perisco P, Colombo S. Canine nodular dermatophytosis (kerion): 23 cases. The authors. Journal compilation 2009; DOI: 10.1111/j.13653164.2009.00749.x.

41. DeBoer DJ. What's new in dermatophytosis?. Proceedings of the 14th Annual Congress of the European Society of Veterinary Dermatology 1997; 15-18.

42. Okabayashi K, Imaji M, Osumi T, Murakami Y, Muruyama $\mathrm{H}$, Kano R, Hhasegawa A, Watanabe T. Antifungal activity of itraconazole and voriconazole aganist clinical isolate from animals with mycoses. Japanese Medical Mycology 2009; 50, 91-94.

43. Aspıroz C, Gilaberte Y, Rezusta A, Boekhout T, Rubio MC. Gentamycin inhibits the growth of Malassezia pachydermatis in culture. Revista Iberoamericana de Micología 2010; 27 (1): 20-21. 